\title{
Gestion Des Dmards Conventionnels Dans La Polyarthrite Rhumatoïde Au Bénin : Quid De La Biothérapie
}

\author{
Zomalhèto Zavier, \\ Assogba Calixte, \\ Dossou-yovo Hilaire,
}

Clinique Universitaire de Rhumatologie du Centre national Hospitalier et Universitaire Hubert Koutoukou Maga de Cotonou, Abomey Calavi (Bénin)

\section{Résumé}

Objectif : Evaluer la gestion et l'efficacité des DMARDs conventionnels chez les patients souffrant de PR au Bénin.

Patients et Méthode : Etude transversale et analytique portant sur les patients suivis pour une PR et traitée par DMARDs. Le diagnostic de PR a été retenu sur la base des critères EULAR/ACR 2010. Les données recueillies ont été analysées grâce au logiciel SPSS17.0

Résultats : 156 patients souffrant de PR ont été évalués. L'âge moyen des patients était de 44,6 $\pm 14,1$ [15-69] ans et le sex- ratio était de 0,14. Le facteur rhumatoïde était présent chez $88 \%$, les anticorps anti-CCP étaient présents chez $69.20 \%$. L'âge moyen de début de la maladie était 32,7 \pm 7.03 [18-50]. La durée moyenne d'évolution était de 10,3 $\pm 7,1$ [0,5-25] ans. Le DAS28 moyen au début était 5,71 \pm 2.04 . Au plan radiologique, le score moyen de Larsen était de 38,55 \pm 16,94. La dose moyenne de Prednisone était de 15 mg/jour. Les DMARDs utilisés étaient : Méthotrexate 15 à 20mg/semaine (150 cas), le léflunomide (6 cas), la salazopyrine 2g/j (27cas), hydroxychloroquine $400 \mathrm{mg}$ / jour (24 cas). A 24 mois, le DAS28 moyen était de 3, 91 $\pm 2,01$ et 23,7\% des patients avaient une PR active.

Conclusion : La disponibilité des DMARDs pose un problème dans notre pays. Leur utilisation rationnelle associée à une faible dose de prednisone permet d'épargner aux patients une biothérapie dont le coût reste hors de la portée des patients de notre pays à faible revenu.

Mots-clés : Polyarthrite Rhumatoïde, Dmards, Corticoïde, Bénin 


\title{
Management Of Synthetic Disease-Modifying Antirheumatic Drugs In Rheumatoid Arthritis In Benin : What About Biological Treatment?+
}

\author{
Zomalhèto Zavier, \\ Assogba Calixte, \\ Dossou-yovo Hilaire,
}

Clinique Universitaire de Rhumatologie du Centre national Hospitalier et Universitaire Hubert Koutoukou Maga de Cotonou, Abomey Calavi (Bénin)

\begin{abstract}
Aim: To evaluate the management and efficacy of conventional DMARDs in RA patients in Benin.

Patients and Method: Transversal and analytical study of patients followed for RA and treated with DMARDs. The diagnosis of RA was selected on the basis of EULAR/ACR 2010 criteria. The data collected were analysed using SPSS17.0 software.
\end{abstract}

Results: 156 RA patients were evaluated. The mean age of the patients was $44.6 \pm 14.1$ [15-69] years and the sex ratio was 0.14 . The mean age of the patients was $44.6 \pm 14.1$ [15-69] and the sex ratio was 0.14 . Rheumatoid factor was present in $88 \%$, anti-CCP antibodies were present in $69.20 \%$. The mean age of disease onset was $10.3 \pm 7.1$ [0.5-25] years. The mean time to progression was $8.9 \pm 7.7$ [1-30]. The mean DAS28 at onset was $5.71 \pm 2.04$ [1-30]. Radiologically, the mean feedback score was $38.55 \pm 16.94$ [1-30]. The mean dose of Prednisone was $15 \mathrm{mg} / \mathrm{day}$. The DMARDs used were: methotrexate $15-20 \mathrm{mg} /$ week (150 cases), leflunomide (6 cases), salazopyrin 2g/day (27 cases), hydroxychloroquine $400 \mathrm{mg} /$ day (24 cases). At 24 months, mean DAS28 was $3.91 \pm 2.01$ and $23.7 \%$ of patients had active RA.

Conclusion: Availability of DMARDs is a problem in our country. Their rational use associated with a low dose of prednisone saves patients from biotherapy, the cost of which remains out of reach for low-income patients in our country.

Keywords: Rheumatoid Arthritis, Dmards, Corticosteroid, Benin 


\section{Introduction}

Les Disease Modifying Anti-Rheumatic Drugs (DMARDs) conventionnels sont utilisés comme traitement de fond dans plusieurs rhumatismes inflammatoires chroniques notamment dans la polyarthrite rhumatoïde (PR) (Felson et al.,1995). Dans la majorité des pays africains où la biothérapie n'est pas disponible du fait de son coût élevé et du risque infectieux, ces traitements conventionnels demeurent incontournables. Certains pays comme l'Afrique du Sud sont très avancés dans l'utilisation de la biothérapie après un échec aux DMARDS conventionnels (Hodkinson et al., 2013). Les DMARDs conventionnels ont un effet symptomatique retardé et un effet sur l'évolution de la maladie, notamment sur la progression radiographique articulaire (Felson,1995). Plusieurs études contrôlées randomisées de moyenne durée ont apprécié l'efficacité clinique des traitements de fond de la PR mais on ne peut dénoter aucun critère clinique ou biologique permettant actuellement de prévoir la réponse d'un patient donné à l'un de ces traitements de fond ni de prédire la survenue des effets indésirables (Cush et al.,2004, Pinals et al., 1981). L'évaluation clinique des traitements de fond de la $\mathrm{PR}$ repose sur plusieurs critères dont les plus utilisés sont ceux basés sur les critères de l'American College of Rheumatology (ACR 20,50,70) et le score d'activité DAS 28 (Disease Activity Score) définissant une rémission pour une valeur inférieure à 2,6 (Prevoo et al., 1996, Van Gestel et al. 1996). La rémission clinique est un objectif majeur dans le traitement de la PR (Cush et al.,2004, Pinals et al., 1981. L'effet structural de ces traitements sur la PR doit être apprécié par des clichés radiographiques annuels ; ce qui est souvent difficilement réalisable dans les conditions de travail en Afrique où le coût élevé des soins ne permet pas une surveillance paraclinique appropriée. Parmi les DMARDs conventionnels, seuls le Méthotrexate, le léflunomide et la sulfasalazine ont montré de façon claire la capacité à réduire la progression radiographique de la PR par rapport à un placebo (Jeurissen et al., 1991, Sharp et al., 2000, Cohen et al., 2001) cette évaluation clinique des DMARDs sur la maladie et les stratégies thérapeutiques y afférents ne sont pas codifiés en Afrique. L'objectif de ce travail a été d'évaluer la gestion et l'efficacité des DMARDs conventionnels dans le traitement de la PR dans un pays n’ayant pas accès à une biothérapie

\section{Patients et Méthode}

Il s'est agi d'une étude transversale descriptive et analytique déroulé du $1^{\text {er }}$ Janvier 2018 au 31 Mars 2020 portant sur les patients suivis dans le service de rhumatologie du Centre National Hospitalier et Universitaire Hubert Koutoukou Maga (CNHU-HKM) de Cotonou et répondant aux critères suivants : 
- Etre suivi dans le service de rhumatologie du CNHU-HKM de Cotonou pour une PR selon les critères EULAR/ACR 2010

- Etre traité par au moins un DMARD conventionnel depuis au moins 2 ans associé ou non à une corticothérapie.

- Avoir honoré tous les rendez-vous de contrôle.

Les patients ayant des contre-indications formelles (hépatopathies, allergies) et qui ne sont pas sous un traitement de fond conventionnel ont été exclus de l'étude.

Le consentement éclairé des patients a été obtenu de même que l'avis du comité d’éthique de la Faculté des Sciences de la Santé.

Ainsi, un recensement exhaustif de tous les cas de PR répondant aux critères d'inclusion a été fait. L'efficacité des divers DMARDs conventionnels a été évalué à travers les paramètres socio-démographiques, cliniques, paracliniques, thérapeutiques et l'index d'activité DAS28. Les données recueillies ont été analysées grâce aux logiciels Epidata et SPSS17.0.

\section{Résultats}

\section{Données socio-démographiques}

Cent cinquante-six (156) patients souffrant de PR et répondant aux critères d'inclusion ont été évalués. L'âge moyen des patients était de 44,6 \pm 14,1 [15-69] et le sex- ratio était de 0,14.

\section{Données cliniques et paracliniques}

Le délai moyen de consultation et de diagnostic était de 36 mois [6120 mois]. Cent onze patients (71,1\%) présentaient des déformations caractéristiques de la PR. L’âge moyen de début de la maladie était 31,7 \pm 7,03 [15-61] ans. La durée moyenne d'évolution était de 10,3 \pm 7,1 [0,5-25] ans

Le facteur rhumatoïde (FR) était présent chez $88 \%$ des patients et les anticorps anti-peptide cyclique citrulliné (anti-CCP), chez 69,2\%.

Au plan radiologique, le score moyen de Larsen des patients à leur inclusion dans l'étude était de 38,55 \pm 16,94.

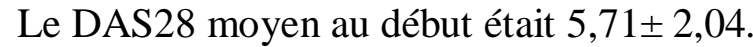

Les données cliniques et paracliniques des patients ont été résumées dans le tableau $n^{\circ} 1$.

Tableau I : Caractéristiques générales des patients

\begin{tabular}{ll}
\hline & Patients (n=156) \\
\hline & Moyenne \pm SD ou n (\%) \\
\cline { 2 - 2 } Age (années) & $44.6 \pm 14.1[17-69]$ \\
Sex-ratio (M/F) & 0.14 \\
Durée d'évolution (années) & $10.3 \pm 7.1[0.5-25]$ \\
Age de début de la maladie (années) & $31.7 \pm 7.03[15-61]$ \\
EVA (mm) & $56.7 \pm 18.3$ \\
\hline
\end{tabular}




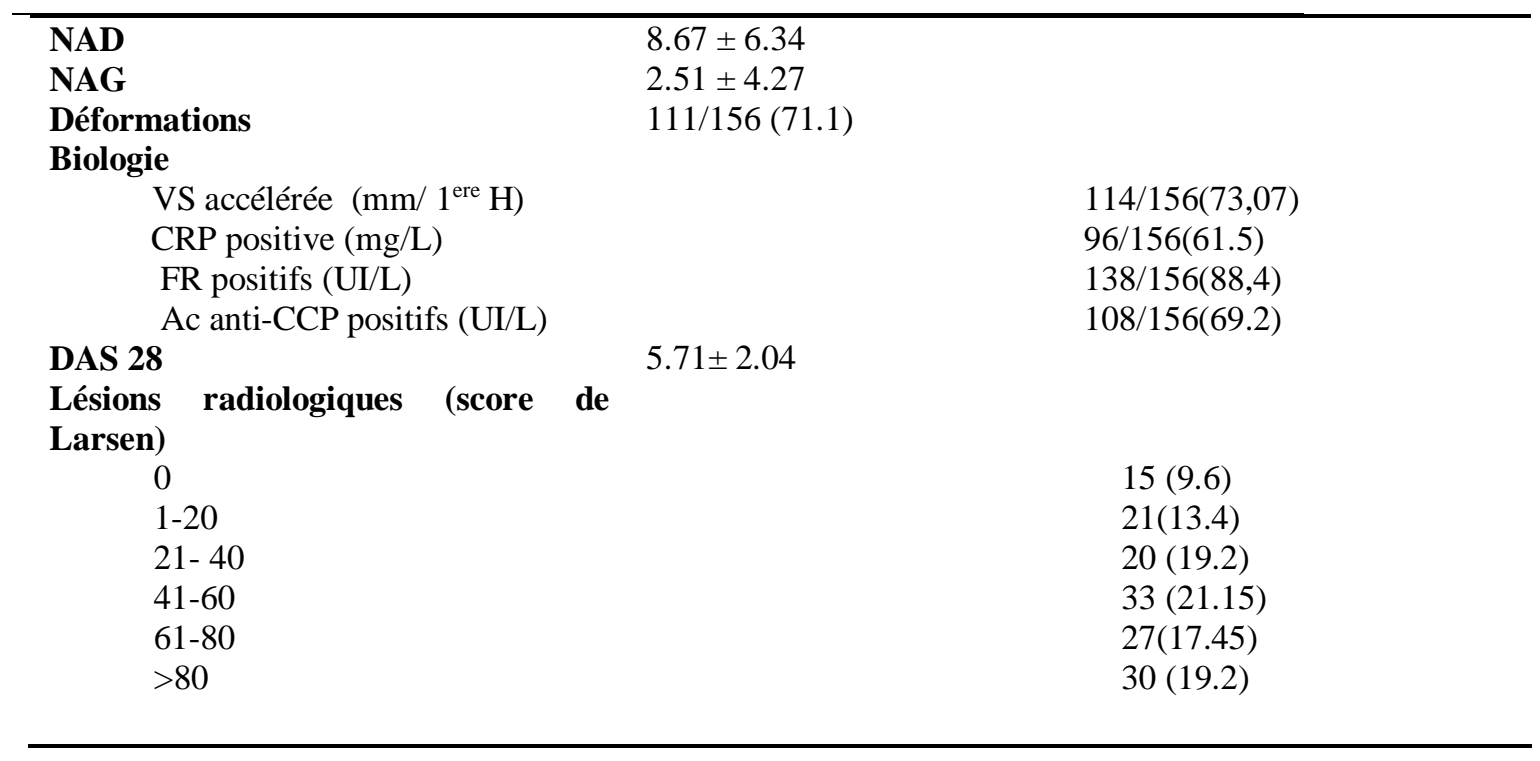

EVA: Echelle Visuelle Analogique, NAD : Nombre d'articulations douloureuses, NAG: Nombre d'articulations gonflées, VS: Vitesse de Sédimentation, CRP: C reactive protein, FR: Facteur Rhumatoïde, AntiCCP:Anticorps Anti-Peptide citrulliné. DAS28: Disease activities score pour 28 articulations

\section{Données thérapeutiques et évolutives}

La dose moyenne de Prednisone était de $15 \mathrm{mg} /$ jour. Les DMARDs utilisés étaient : le méthotrexate avec une dose maximale de 20mg/semaine (150 cas), le léflunomide à la dose de $20 \mathrm{mg} / \mathrm{j}$ (6 cas), la salazopyrine à la dose de 2g/j (27 cas), hydroxychloroquine $400 \mathrm{mg} /$ jour (24 cas). Aucune biothérapie n’était disponible dans l'arsenal thérapeutique

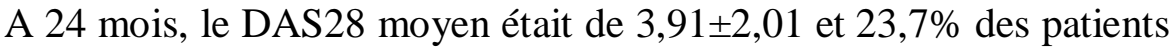
avaient une PR active. Par ailleurs, plus de la moitié des patients avaient encore une corticothérapie. Le tableau $n^{\circ} 2$ ainsi que les figures $n^{\circ} 1$ et 2 rendent comptent des données thérapeutiques et évolutives des patients suivis.

Tableau II : Gestion des DMARDs

\begin{tabular}{lllllll}
\hline & Début & $\mathbf{3}$ mois & $\mathbf{6}$ mois & $\mathbf{1 2}$ mois & $\mathbf{1 8}$ mois & $\begin{array}{l}\mathbf{2 4} \\
\text { mois }\end{array}$ \\
\hline Méthotrexate & 156 & 156 & 150 & 144 & 144 & 135 \\
Léflunomide & 00 & 00 & 06 & 6 & 06 & 06 \\
Salazopyrine & 00 & 00 & 00 & 21 & 27 & 27 \\
Hydrohychloquine & 00 & 00 & 00 & 00 & 15 & 24 \\
Biothérapie & 00 & 00 & 00 & 00 & 00 & 00 \\
\hline
\end{tabular}


Nombre de patients

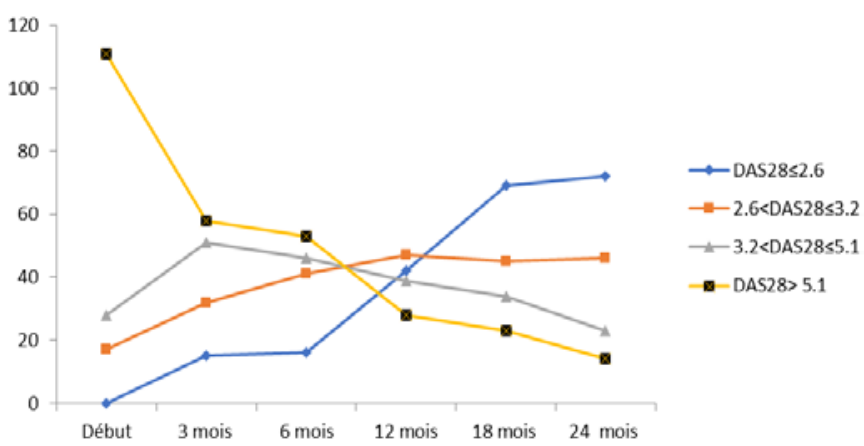

Mois

Figure 1 : Evolution du DAS 28 des patients durant les 2 premières années

\section{Nombre de}

patients

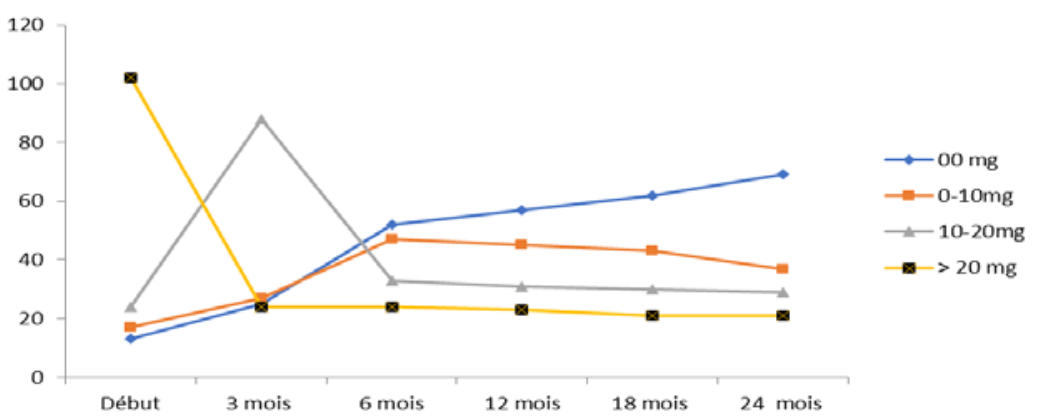

Mois

Figure 2: Gestion de la corticothérapie durant les 2 premières années

\section{Discussion}

Le long délai de consultation, lié au nombre très infirme de rhumatologues au Bénin (6 rhumatologues pour 10 millions d'habitants) et à la méconnaissance de la maladie, occasionne le retard diagnostique observé. Ce retard a pour conséquence qu'une grande majorité des patients présentant des lésions radiographiques au moment du diagnostic responsables d'une rémission retardée ou absente. Au plan thérapeutique, il existe une grande difficulté à l'acquisition de traitements de fond conventionnels. En dehors du méthotrexate per os dont la disponibilité est émaillée des épisodes de rupture sur le territoire national et même dans la sous-région avoisinante, les autres DMARDs conventionnels ne sont pas présents malgré la lutte des prescripteurs pour l'inscription de ces médicaments sur la liste des médicaments essentiels. La stratégie thérapeutique pour la gestion des patients suivis pour la polyarthrite rhumatoïde est donc conditionnée par la question de disponibilité des DMARDs qui dans la majorité des cas est commandée en l'Europe par les patients. 
Le méthotrexate, conformément aux diverses recommandations sur la prise en charge de la PR a été le traitement de fond de choix de la polyarthrite rhumatoïde des patients suivis (Jeurissen et al., 1991, 2000, Cohen et al., 2001). En effet, il reste et demeure la pierre angulaire du traitement de la polyarthrite rhumatoïde (Banwarth et al., 1994, Edno et al., 1995, Berthelot et al., 2001). Il s’agit d'un antimétabolite analogue de l'acide folique qui inhibe la dihydrofolate réductase en se liant de façon réversible à cette enzyme. Il constitue actuellement le traitement de référence de la PR ("Gold Standard") à l’échelon mondial (Berthelot et al., 2001, Sany, 2003).

Par ailleurs, l'efficacité clinique du méthotrexate a été démontrée au cours de nombreuses études contrôlées en double aveugle contre placebo et au cours d'études comparatives avec les DMARDs conventionnels et la biothérapie notamment les anti-TNFa. Cette efficacité clinique après deux ans était du même ordre que celle obtenue avec les anti-TNF en monothérapie bien que l'obtention soit moins rapide et que les anti-TNF gardent néanmoins un avantage net sur le contrôle de la progression structurale (Hazlewood et al., 2016, Bathon et al., 2000, Breedveld et al., 2004, Pincus et al., 2003). Quant à la posologie, elle va de 7,5mg à $20 \mathrm{mg}$ par semaine chez nos patients. Les tentatives d'une posologie au-delà de 20 mg / semaine ont été soldées par des effets secondaires digestifs et des cytolyses hépatiques ayant conduit à la diminution des doses. Les réunions d'experts retiennent une posologie initiale conseillée du méthotrexate dans la $\mathrm{PR}$ de 10 à $15 \mathrm{mg} / \mathrm{semaine}$ avec augmentation progressive par palier de $5 \mathrm{mg} / \mathrm{mois}$ jusqu'à une dose maximale de $20 \mathrm{mg}$ voire $30 \mathrm{mg}$ par semaine, en fonction du poids et de la tolérance, chez les sujets insuffisamment répondeurs (Hazlewood et al., 2016, Visser et van der Heijde,2009). Près de 85 \% des patients gardait une PR faiblement ou modérément active après un an de traitement par méthotrexate ; ce qui est bien supérieure aux taux retrouvés par d’autres auteurs. En effet, le pourcentage de patients répondant au méthotrexate varie de 50 à $60 \%$. Une rémission selon les critères de l'ACR est rapportée dans 6,8 à 14 \% des cas [Prevoo et al., 1996, Berthelot et al., 2002, Hazlewood et al., 2016).

Devant les difficultés locales liées à la non disponibilité des formes injectables de méthotrexate (sous-cutanée et intramusculaire) et surtout de la biothérapie pour diverses raisons, les stratégies de combinaisons ou de substitution du méthotrexate ont pris une place importante dans la stratégie de gestion de nos patients avec une efficacité plus ou moins acceptable (rémission à plus de 75\%). Plusieurs études ont prouvé l'efficacité (clinique et structurale) de ces associations dans le traitement de la PR avec des résultats comparables à ceux du méthotrexate en monothérapie et celle de l'association biomédicament- méthotrexate (Hazlewood et al., 2016).

Plus de la moitié des patients avait une corticothérapie <10mg ou n’étaient plus sous corticoïdes au bout de 2 ans de suivi des patients. L’épargne 
cortisonique est un critère important de l'efficacité du traitement de fond. Plusieurs études ont démontré cette possibilité de réduction ou d'arrêt des corticoïdes sous DMARDs. Le pourcentage de patients pouvant interrompre la corticothérapie varie de 12,6 à 33,3\% avec une dose nulle ou < 7,5 mg/j. De façon générale, l’épargne cortisonique doit être atteinte le plus rapidement possible avec une gestion adaptée à l'état clinique et à la disponibilité des traitements de fond (Berthelot et al., 2002, Bressolle et al., 1998, Wolde et al., 1996, Beltrametti et al., 2016).

Cependant, le nombre non négligeable de patients sous corticothérapie à dose élevée malgré les associations de traitements de fond conventionnels, pose le problème de l'inaccessibilité du traitement biologique pouvant aider à contrôler la maladie. La prise en charge efficiente de ces patients passe nécessairement par une politique de réduction des coûts des biothérapies permettant leur accessibilité aux patients non contrôlés par les DMARDS conventionnels

\section{Conflit d'intérêt}

Aucun

\section{Conclusion}

La rémission de la $\mathrm{PR}$ nécessite l'utilisation des DMARDs dont la disponibilité pose un problème dans certains pays de l'Afrique noire. Le méthotrexate reste cependant la pierre angulaire du traitement chez les patients avec une bonne efficacité. Il persiste cependant près du quart des patients chez qui une association de DMARDs conventionnels et de corticothérapie au long cours s'avère indispensable du fait de l'absence d'une biothérapie hors de la portée des patients. Il urge de mener une politique de réduction de coût et d'assurance maladie universelle pouvant faciliter l'accessibilité du traitement biologique aux patients.

\section{References:}

1. Felson DT, Anderson JJ, Boers M, C Bombardier, D Furst, C Goldsmith, L M Katz, R Lightfoot Jr, H Paulus, V Strand. American College of Rheumatology preliminary definition of improvement in rheumatoid arthritis. Arthritis Rheum 1995 ; 38 : 722-735.

2. Hodkinson B, Van Duuren E, Pettipher C, Kalla A. South African recommendations for the management of rheumatoid arthritis: an algorithm for the standard of care in 2013. S Afr Med J. 2013 Jun 14;103(8 Pt 2):576-85.

3. Cush JJ. Safety overview of new disease-modifying antirheumatic drugs. Rheum Dis Clin N Am 2004 ; 30 :237-55. 
4. Pinals RS, Masia T, Larsen RA. Preliminary criteria for clinical remission in rheumatoid arthritis. Arthritis Rheum 1981 ; 24 : 13081315.

5. Prevoo ML, van Gestel AM, van T Hof MA, van Rijswijk MH, van de Putte LB, van Riel PL. Remission in a prospective study of patients with rheumatoid arthritis. American Rheumatism Association preliminary remission criteria in relation to the disease activity score. Br J Rheumatol. 1996 ;35:1101-1105.

6. van Gestel AM, Prevoo MLL, vant ${ }^{\text {ee }}$ Hof MA, van Rijswijk MH, van de Putte LBA, van Riel PLCM. Development and validation of the European League Against Rheumatism response criteria for rheumatoid arthritis. Arthritis Rheum 1996 ; 39 : 34-40.

7. Jeurissen MEC, Boerbooms AMT, Van de Putte LB, Doesburg WH, Lemmens AM. Influence of methotrexate and azathioprine on radiologic progression in rheumatoid arthritis. Ann Int Med 1991 ; 114 : 999-1004.

8. Sharp JT, Strand V, Leung H, Hurley F, Loew-Friedrich I. Treatment with leflunomide slows radiographic progression of rheumatoid arthritis: results from three randomized controlled trials of leflunomide in patients with active rheumatoid arthritis. Arthritis Rheum 2000 ; 43 : 495- 505.

9. Cohen S, Cannon GW, Schiff M, A Weaver, R Fox, N Olsen, D Furst, J Sharp, L Moreland, J Caldwell, J Kaine, V Strand. Two-year, blinded, randomized, controlled trial of treatment of active rheumatoid arthritis with leflunomide compared with methotrexate. Arthritis Rheum 2001 ; 44 :1984-92

10. Banwarth B, Labat L, Moride Y, Schaeverberke T. Methotrexate in rheumatoid arthritis. An update. Drugs 1994 ; 47 : 25-50.

11. Edno L., Bressolle F., Gomeni R., Bologna C., Sany J., Combe B. Total and free methotrexate pharmacokinetics in rheumatoid arthritis patients. Therapy Drug Monitoring, 1995, 18, 128-134.

12. Berthelot JM, Combe B. Efficacité, tolérance et maintien du méthotrexate dans le traitement des polyarthrites rhumatoïdes. Revue du Rhumatisme 2002 ; 69 (suppl.2) : 34-43.

13. Sany J. Polyarthrite Rhumatoïde de leadulte. John Libbey ed., Paris, 2003, pp 171-272.

14. Hazlewood GS, Barnabe C, Tomlinson G, Marshall D, Devoe D, Bombardier C. Methotrexate monotherapy and methotrexate combination therapy with traditional and biologic disease modifying antirheumatic drugs for rheumatoid arthritis: abridged Cochrane systematic review and network meta-analysis. BMJ 2016; 353: i1777 
15. Bathon JM, Martin RW, Fleixchmann RM, J R Tesser, M H Schiff, E C Keystone, M C Genovese, M C Wasko, L W Moreland, A L Weaver, J Markenson, B K Finck. A comparison of Etanercept and methotrexate in patients with early rheumatoid arthritis. N. Engl. J. Med., 2000 ; 343 : 1586-1593.

16. Breedveld F, Kavanaugh AF, Cohen SB, Pavelka K, van Vollenhoven R, Pérez JL. Early treatment of Rheumatoid Arthritis with adalimumab plus methotrexate vs adalimumab alone or methotrexate alone: the PREMIER study. Arthritis Rheum 2004; 50:4096 (LBP 5).

17. ) Pincus T, Yazici Y, Sokka T, Aletaha D, Smolen JS. Methotrexate as the "anchor drug" for the treatment of early rheumatoid arthritis. Clin Exp Rheumatol. 2003; 21(5 Suppl 31):S179-185

18. Visser K, van der Heijde D. Optimal dosage and route of administration of methotrexate in rheumatoid arthritis: a systematic review of the literature. Ann Rheum Dis 2009;68(7): 1094-1099.

19. Bressolle F., Bologna C., Kinowski J.M., Sany J., Combe B. Effects of moderate renal insufficiency on pharmacokinetics of methotrexate in rheumatoid arthritis patients. Annals of Rheumatic Diseases 1998 ; 57 :110-3

20. Wolde ST, Breedveld FC, Hermans J, Vandenbroucke JP, van de Laar MA, Markusse HM, Janssen M, van den Brink HR, Dijkmans BA. Randomised placebo-controlled study of stopping second-line drugs in rheumatoid arthritis. Lancet 1996 ; 347 : 347-352.

21. Beltrametti SP, Ianniello A, Ricci C. Chronotherapy with low-dose modified release prednisone for the management of rheumatoid arthritis: a review. Ther Clin Risk Manag 2016 ; 12 :1763-76 